\title{
Weak equivalence in the balance
}

If the weight, but not the inertial mass, of an antiproton should differ from that of a proton, there would be important consequences for the theories of relativity and of particles. But informed opinion is sceptical of the possibility.

THE idea that the weights of protons and antiprotons may differ from each other is not nearly the arcane speculation it might seem. Indeed, it is not just a speculation, but the focus of some considerable interest among experimentalists. For one thing, there is a proposal directly to measure the difference of the weight of the two particles now being implemented at the European high-energy physics laboratory, CERN, at Geneva.

The neatness of the experiment is almost a sufficient justification of it, which may (as will emerge) be just as well. The plan is to cool antiprotons from the LEAR storage ring to a few degrees kelvin, and then to launch them vertically upwards in a drift tube and to measure the time it takes for them to reach a predetermined height. There is even talk of carrying out an essentially static measurement of the weight (or gravitational attraction) by letting atoms of anti-hydrogen (a positron bound to an antiproton, yet to be manufactured) fall freely under gravity, which would avoid the trouble to be expected from stray electric fields in a measurement of free charged particles.

But why? The inertial masses of a particle and its antiparticle should be identical, so that any reasonable theory of gravitation, such as general relativity, should enjoin equal weights: weigh one, and you have weighed the other. But that is not quite the end of the story, at least if hopes of winning a quantum theory of gravitation are taken seriously (as they must be if there is ever to be a unified theory of all the forces between particles). In principle, at least, quantum gravity could allow the weights of the proton and antiproton to differ.

But how? E. D. Adelberger, B. R. Heckel, C. W. Stubbs and Y. Su of the Washington University, St Louis, give a cogent explanation in the course of arguing that the observations already made and recorded provide as good a test of the reality of a weight difference as the planned experiments are likely to provide (Phys. Rev. Lett. 66, 850; 18 February 1991).

If the general theory of relativity is ever consistently quantized along the lines of electrodynamics, for example, the gravitational forces between different particles will no doubt be mediated by other particles just as the forces of electrodynamics are mediated by photons, which are massless particles whose spin is 1 . Indeed, there is already a name for these particles, which are called 'gravitons', presumably (like photons) with no mass. Moreover, the spin of the graviton must be 2, or twice that of the photon, which ensures that gravitational forces are always attractive.

The snag is that, in quantum mechanics, hardly any state is ever completely pure in the strict sense of that word; in this case, the spin-2 gravitons may well coexist with gravitons of spin 1 and spin 0 . And then the universally attractive forces of gravitation would be mixed with repulsive forces mediated by the spin-1 gravitons and also with attractive forces arising from the spin-0 particles.

Recollections of the excitement over the 'fifth force' of a few years back, far from being out of place, are accurately pertinent, and for two reasons. First, the fifth force was also supposed to be mediated by particles of integral spin (or bosons). Second, the spate of experimental measurements provoked by that excitement may have tightly constrained the extent to which materials of different chemical composition are differently affected, but none of them has excluded the possibility that particles and antiparticles may be differently affected by long-range forces resembling those of gravitation.

There is an even simpler way of putting the conundrum. It is fair to hold that the greatest of Newton's discoveries was the implication that inertial mass (defined as the ratio of the force on an object to the acceleration it produces) and gravitational mass are strictly equivalent. The relativistic equivalent of that principle, called the 'weak equivalence principle', is that one must also take account of whatever internal energy an object may have by the familiar $E=m c^{2}$ rule. So differences between the weight of protons and antiprotons would, on the face of things, imply violations of the weak equivalence principle.

In the same issue of Physical Review Letters, Richard J. Hughes and Michael $\mathrm{H}$. Holzscheiter, from the Los Alamos National Laboratory, argue that 'CPT' symmetry, which is an article of many faiths, may alternatively be violated $(66,854 ; 18$ February 1991). In this rubric, $C$ stands for the operation of charge conjugation, which replaces each particle by its antiparticle (whenever possible), $\mathrm{P}$ stands for the parity operation (which is space inversion) and $\mathrm{T}$ stands for time inversion. Or, turning the argument around, if CPT symmetry is exact, and if antiprotons weigh differently from protons, the weak equivalence principle must be violated.

What do existing data say? Adelberger $e t$ $a l$. argue that data such as those accumulated during the hunt for the fifth force (including a more generally directed measurement of their own intended to measure the differences (if any) of the gravitational acceleration of pairs of materials such as beryllium and copper) exclude the possibility that quantum gravity may give antiprotons and protons different weights or, more accurately, they say that any gravitational interaction caused by spin-1 gravitons is unlikely to amount to more than one part in 100 million of conventional gravity.

They go on to exclude the theoretical possibility that the effects of spin-1 and spin-0 gravitons may cancel in protons, but not in antiprotons, by insisting that cancellation would not be possible for all pairs of materials and for all the physical dimensions (from a metre to some millions of kilometres) over which the equivalence principle has been verified.

Hughes and Holzscheiter are similarly despondent at the prospects of finding that antiprotons weigh differently from protons. For one thing, they note, there have already been measurements of the cyclotron frequencies of protons and antiprotons that effectively restrict the difference of their weight to less than one part in 1,000, a tighter limit that than likely to be attained in the first version of the experiment planned at CERN. They go on to argue that more stringent limits will quickly be possible as the effectiveness of electromagnetic traps for single particles improves.

So, on the face of things, the planned experiments are unlikely to yield surprises when the first results emerge, perhaps two years from now. Yet that is not a reason why they should be abandoned. For one thing, they are neat. For another, even if other than a null result would be a violation of the weak principle of equivalence which has been amply verified in other circumstances, there are the strongest reasons why principles of such importance should be tested in circumstances as varied as they can be.

Hughes and Holzscheiter raise a further question of literally cosmic significance: if gravitons are not strictly massless, but have a small mass (as is canvassed for some neutrinos), the range of gravitional forces will not be infinite, but may still be of galactic or cluster scale, suggesting that the most productive experiments will be those comparing cyclotron frequencies, say, here and at the distant reaches of the Universe. There is a challenge for the experimenters.

John Maddox 